\title{
Experimental study on active structural acoustic control of rotating machinery using rotating piezo-based inertial actuators
}

\author{
G. Zhao ${ }^{\mathrm{a}, *}$, N. Alujević ${ }^{\mathrm{a}}$, B. Depraetere ${ }^{\mathrm{b}}$, G. Pinte $^{\mathrm{b}}$, J. Swevers ${ }^{\mathrm{a}}$, P. Sas ${ }^{\mathrm{a}}$ \\ ${ }^{a}$ KU Leuven, Production Engineering, Machine Design and Automation (PMA) Section, Celestijnenlaan $300 b$ - box 2420,3001 Heverlee, \\ Belgium \\ ${ }^{\mathrm{b}}$ FMTC, Flanders' Mechatronics Technology Centre, Celestijnenlaan 300d - box 4027, 3001 Heverlee, Belgium
}

\section{A R T I C L E I N F O}

\section{Article history:}

Received 18 July 2014

Received in revised form

30 January 2015

Accepted 4 March 2015

Handling Editor: J. Lam

Available online 6 April 2015

\begin{abstract}
A B S T R A C T
In this paper, two Piezo-Based Rotating Inertial Actuators (PBRIAs) are considered for the suppression of the structure-borne noise radiated from rotating machinery. As add-on devices, they can be directly mounted on a rotational shaft, in order to intervene as early as possible in the transfer path between disturbance and the noise radiating surfaces. A MIMO (Multi-Input - Multi-Output) form of the FxLMS control algorithm is employed to generate the appropriate actuation signals, relying on a linear interpolation scheme to approximate time varying secondary plants. The proposed active vibration control approach is tested on an experimental test bed comprising a rotating shaft mounted in a frame to which a noise-radiating plate is attached. The disturbance force is introduced by an electro-dynamic shaker. The experimental results show that when the shaft spins below $180 \mathrm{rpm}$, more than a $7 \mathrm{~dB}$ reduction can be achieved in terms of plate vibrations, along with a reduction in the same order of magnitude in terms of noise radiation.
\end{abstract}

(c) 2015 Elsevier Ltd. All rights reserved.

\section{Introduction}

Noise radiation from structural housing in rotating machinery is a common problem in many industrial applications such as gearboxes, compressors, electric motors etc. In these cases, vibrations of rotating elements, which are transmitted though the bearings to the noise radiating surfaces such as the machine frame, are often the major noise source. In order to reduce the received noise level, techniques such as sound absorption based insulation, including encapsulation, can be used to interrupt the airborne sound transmission from the machine to the environment. These passive sound control techniques would typically be used to deal with noise at higher frequencies. At lower frequencies, where the acoustic wavelength is much larger than the maximum permissible thickness of the insulation layers, active noise control strategies can be considered instead [1-4]. These however become more complicated and more expensive, or alternatively less efficient, if the size of the enclosure where the sound is controlled [3] is comparatively large. Many error sensors and control actuators are necessary for good control performance, and in fact the total length of the wiring to connect peripheral units to the centralised controller can become a limitation [4]. In such situations it could be preferred to directly reduce the vibroacoustic response of the noise radiating surfaces. This can be done by applying control forces on the surfaces [5,6,11-17]

\footnotetext{
* Corresponding author.

E-mail address: nudt.guoying@gmail.com (G. Zhao).
} 
or isolating the transmission force to the surfaces [18-25]. In case forces are applied directly to the noise radiation surfaces, passive tuned mass dampers [7-10], inertial shakers [11-13], reactive actuators [14] or piezoelectric patches [15-17] are often employed to produce the control forces. However, this approach may become cumbersome and expensive for large and complex systems which have many radiating surfaces.

On the other hand, in the active vibration isolation approach it is attempted to block the transmitted vibrations in the structural transfer paths before they reach the noise radiating surfaces. This may yield a control system that is less complex in case there are concentrated bottlenecks in the vibration transmission paths. With rotating machinery such concentrations typically occur in bearings that support revolving shafts. Several studies based on this approach have been published recently. Rebbechi et al. [19] proposed to integrate two pairs of magnetostrictive actuators into a double row bearing, which is mounted on the input shaft next to the input pinion, with the aim of actively isolating the vibration transmitted from the shaft to the housing. A reduction of $20-28 \mathrm{~dB}$ can be obtained in the housing vibration at the fundamental gear mesh frequency. Pinte et al. [20] and Stallaert et al. [21] adopted a similar approach, but used two piezoelectric actuators instead, which are perpendicularly mounted onto one of the support bearing locations in order to limit the force transmitted from the shaft to the housing. Instead of placing the actuator in series with the original bearing, Guan et al. [22] and Li et al. [23] proposed to mount the control actuator in parallel, directly on the shaft via an additional bearing (active shaft transverse vibration control). In such a case, the system suspension stiffness is not influenced by the introduction of the actuator. Chen and Brennan [24] investigated an inertial actuator control concept, where three magnetostrictive inertial shakers are positioned tangentially at $120^{\circ}$ intervals on the gear body, in order to suppress the gear vibrations at the source. The above mentioned actuation concepts for the suppression of gearbox housing vibrations are theoretically compared by Guan et al. [25]. In this theoretical study, the actuation effort, control robustness and implementation costs are taken into account as the comparison criteria for four different actuation concepts. The shaft transverse vibration active control approach appeared to be the best compromise regarding the required amplitude of the control force below $500 \mathrm{~Hz}$ while also yielding a reasonable control robustness and a limited implementation cost.

In this study, an axisymmetric piezo-based rotational inertial actuator, which can be installed directly on the rotating shaft as an add-on device, is proposed and studied experimentally. The benefit of this add-on approach is that the machine stiffness is not affected as is the case with for example active bearings. Another advantage is the relative ease of implementation in a practical setting as no major structural modification is required. Furthermore, the active element is not in a critical path of the machine such that a possible failure of the piezoelectric element does not necessarily affect the functionality of the machine.

One of the aims of this paper is to investigate whether or not it is feasible to suppress tonal structural borne noise/ vibration by attaching PBRIAs directly onto a slowly rotating shaft. The control strategy is based on the filtered reference least mean squares (FxLMS) algorithm [1,2]. Such an algorithm has been adapted in this study for the use on rotating machine applications by utilising an interpolation scheme for time varying secondary plants. The design of the PBRIA is briefly covered in Section 2, along with the experimental test bed used to evaluate its performance. The implementation of the FxLMS control algorithm is discussed, in Section 3, followed by a presentation of the achieved results in Section 4.

\section{Design of the piezo-based rotating inertial actuator}

Fig. 1 shows the developed prototype of the piezo-based rotating inertial actuator. The idea behind the design of the PBRIA is to use a piezoelectric actuator to introduce a force between a rotating element (e.g. the shaft) and a ring-shaped mass rotating together with the shaft. By accelerating the ring-shaped mass, compensating forces can be generated on the shaft. The Piezomechanik HPSt 150/20 piezoelectric stack actuator is used. Although the developed PBRIA has a sufficient force capacity to compensate the disturbances on the test bed in the frequency range of interest $(200-1000 \mathrm{~Hz})$, it is acknowledged that in industrial applications, where excitation forces are larger, longer piezoelectric actuators or heavier proof mass should be used to generate the required compensating force.

In order to eliminate the need for an additional fixture mechanism, the inner collar, as shown in Fig. 1, is split. Moreover, the splits are offset in order to prevent stress concentration on the piezoelectric actuator. Note that the maximum applicable negative voltage for the piezoelectric stack actuator is much smaller than the maximum applicable positive voltage so that

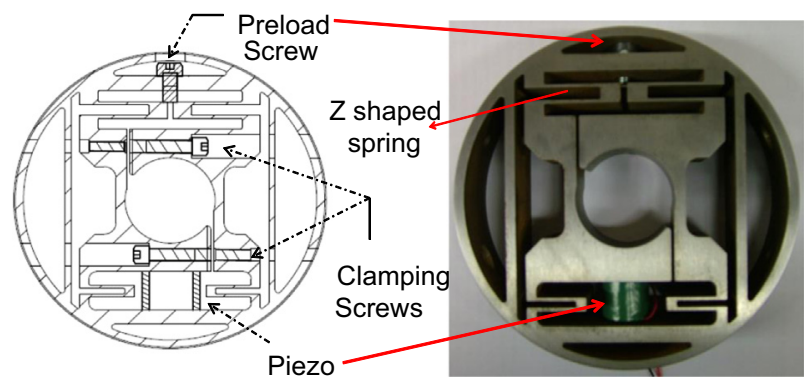

Fig. 1. The developed piezo-based rotating inertial actuator. 
the piezoelectric stack actuator cannot withstand a high level of tension force. In practice, this issue can be solved by applying a preload force to the piezoelectric actuator. In this design, the piezoelectric actuator is preloaded by pre-stressing two Z shaped springs on the opposite side, such that it is capable of applying bi-directional (push/pull) forces. A cap screw is then used to tighten the $\mathrm{Z}$ shaped springs and a pre-stress of approximately $250 \mathrm{~N}$ is applied to the piezoelectric element.

The proof mass (outer ring) of the PBRIA is suspended by four $Z$ shaped springs. These springs are designed to realise a low translational stiffness in one radial direction (vertical direction in Fig. 1) while guaranteeing a high stiffness in the other radial direction (horizontal direction in Fig. 1) as well as high torsional stiffness. A high torsional stiffness is designed in order to avoid excessive bending stress for the piezoelectric actuator in the low frequency range.

The actuation performance of the current design is assessed by measuring its blocked force, which is defined as the force that the actuator generates when its attachment point is constrained not to move [26]. Fig. 2 shows the set-up made for the measurements of the blocked force. The inner ring of the PBRIA is fixed to a heavy block mass through an impedance head ( $P C B$ 288D01) which is used to measure the force generated by the PBRIA projected in the vertical direction. The heavy block approximates a rigid boundary (blocked mass) and therefore the force measured by the impedance head can be considered as the blocked force. A SCADAS III system running LMS Test.lab is used to measure the frequency response function (FRF) of the transfer function between the voltage applied to the piezoelectric actuator and the generated force.

The blocked force FRF (between the force and the input voltage) is measured in the frequency range $0-2 \mathrm{kHz}$, as shown in Fig. 3. In general, the maximum of the response function occurs at about $1300 \mathrm{~Hz}$, which corresponds to the translational mode of the PBRIA. Besides this peak, a sequence of peaks and dips are presented in the frequency ranges: (1) between $500 \mathrm{~Hz}$ and $700 \mathrm{~Hz}$ and (2) between 1400 and $1600 \mathrm{~Hz}$ in the measured FRF, which are due to the dynamics of the measurement set-up. For the frequencies below the fundamental frequency, the magnitude response reduces and the phase response tends to be $180^{\circ}$, which indicates that the blocked force at the inner ring of the PBRIA is out of phase with the driving voltage signal. Around $1300 \mathrm{~Hz}$, the phase of the blocked force FRF undergoes a $180^{\circ}$ phase-lag such that the blocked force is in phase with the driving signal at higher frequencies and the magnitude response is approximately flat up to $2 \mathrm{kHz}$.
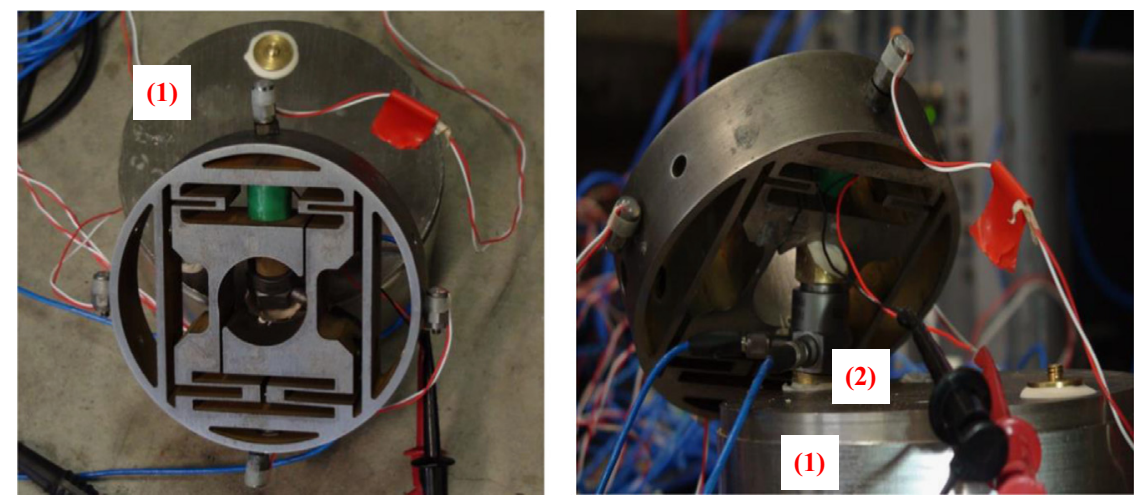

Fig. 2. The measurement set-up for the blocked force: (1) base mass; (2) impedance head.
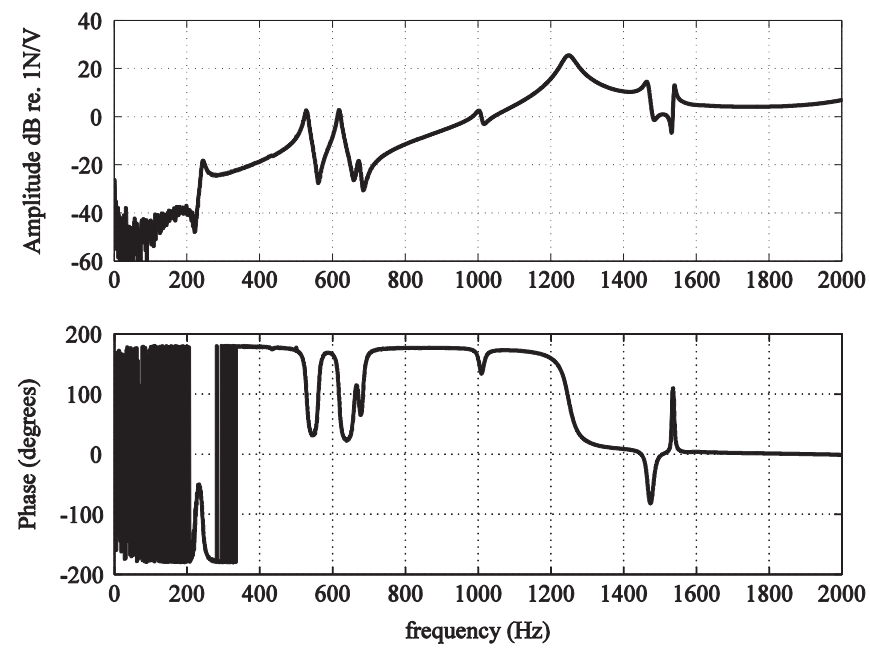

Fig. 3. The measured frequency response between the blocked force and the driving voltage. 
It can be seen that the control force which can be applied by the PBRIA only starts to be significant when the frequency is above $200 \mathrm{~Hz}$.

Therefore, the fundamental resonant frequency of the PBRIA should be carefully designed, which ideally needs to be near or less than the excitation frequency in order to have a constant and sufficient force excitation for low frequencies, see for example [27] where collocated control with direct velocity feedback has been considered. The proposed PBRIA utilises a piezoelectric stack actuator which yields a high piezoelectric coupling coefficient ( $d_{33}$ piezoelectric effect) but also associates a high fundamental resonance frequency around $1300 \mathrm{~Hz}$. This high resonance frequency of the PBRIA may preclude its application in the low frequency range. In order to lower the resonant frequency of the PBRIA, piezoelectric beams instead of piezoelectric stacks could be used in conjunction with bending beams to accelerate the outer ring mass with a $d_{31}$ piezoelectric effect, see for example [10]. However, one should bear in mind that the piezoelectric coupling coefficient for the $d_{31}$ mode is reported to be approximately 2 times smaller than the $d_{33}$ mode [28], which means it may consume more energy to exert the same control force. In addition, the fundamental resonance frequency of the PBRIA should be much larger than the rotating speed in order to maintain its functionality as an inertial actuator. Therefore, the actuating mechanism of choice for a PBRIA has to combine the above three considerations. The developed PBRIA has been tested to be able to excite most structural vibration modes of the test bed (the test bed will be introduced in the following paragraph) in the frequency range of interest [29], which indicates the PBRIA has control authority over the concerned frequency range.

A representative set-up for rotating machinery is constructed in order to demonstrate the feasibility of the proposed ASAC control approach, which is shown in Fig. 4. The test bed consists of a shaft which is mounted in a frame by a cylindrical bearing at one side and a double angular contact ball bearing at the other side. This latter bearing is mounted in a ringshaped module in which two piezoelectric sensors are installed to measure the transmitted forces between the shaft and the frame. Close to this bearing, two PBRIAs are perpendicularly mounted on the shaft such that control forces can be generated in all directions while the shaft rotates. An electric motor is connected to the shaft to drive the shaft. A steel plate with dimensions $40 \mathrm{~mm} \times 30 \mathrm{~mm} \times 2 \mathrm{~mm}$ (length $\times$ height $\times$ width) is connected to the frame using two square U-beam profiles and acts as a noise radiator. The dimensions of the different parts are chosen such that the test bed has a representative dynamic and acoustic behaviour for industrial rotating machinery such as gearboxes [21]. A slip ring is mounted on the shaft close to the PBRIAs to provide electrical connections to the non-rotating controller.

In rotating machinery, the disturbance force can be for example caused by unbalance, misalignment of the shaft, or a mechanical process such as meshing of gears in gearboxes and forces generated by a rotational pump or compressor. In the employed set-up, the disturbance force is artificially generated by an electro-dynamic shaker which is connected to the shaft via an additional bearing. With the additional bearing, it is possible to introduce arbitrary disturbance forces during the rotation of the shaft.

A number of sensors are installed on and around the test bed to measure the performance of the proposed control approach. The layout of the sensor configuration is shown in Fig. 5, where two accelerometers are used to measure the structural vibrations, one microphone is used to register the noise level in front of the plate at a distance of approximately $30 \mathrm{~cm}$, and one force gauge, placed between the bearing close to the PBRIA and the frame, is used to record the transmitted force. Considering now the accelerometers, one of them is placed on the frame measuring vibrations in $x$ and $y$ axes, while the other one is mounted in the middle of the plate measuring only vibrations along the $x$ axis. The $x$ and $y$ axes represent the directions that are parallel and perpendicular to the disturbance line of action, as indicated in Fig. 5. Hereafter, $x$ direction refers to the horizontal direction and $y$ direction refers to the vertical direction. The frame vibration along the $y$ axis (the vertical frame acceleration) and plate vibrations along the $x$ axis (the horizontal plate acceleration) are chosen as the error signals for the active controller in the experiments of this study.

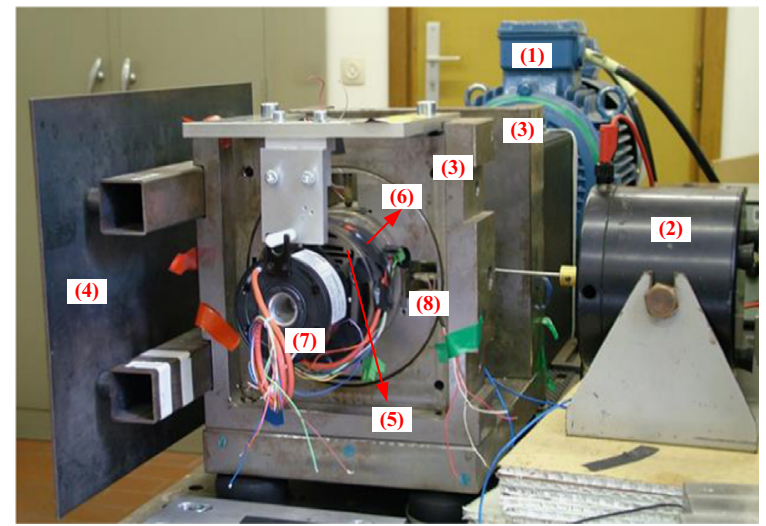

Fig. 4. The experimental set-up of the test bed: (1) Motor; (2) Disturbance shaker; (3) Frame; (4) Noise radiating plate; (5) PBRIA 1; (6) PBRIA 2; (7) Slip ring; (8) Force sensor. 


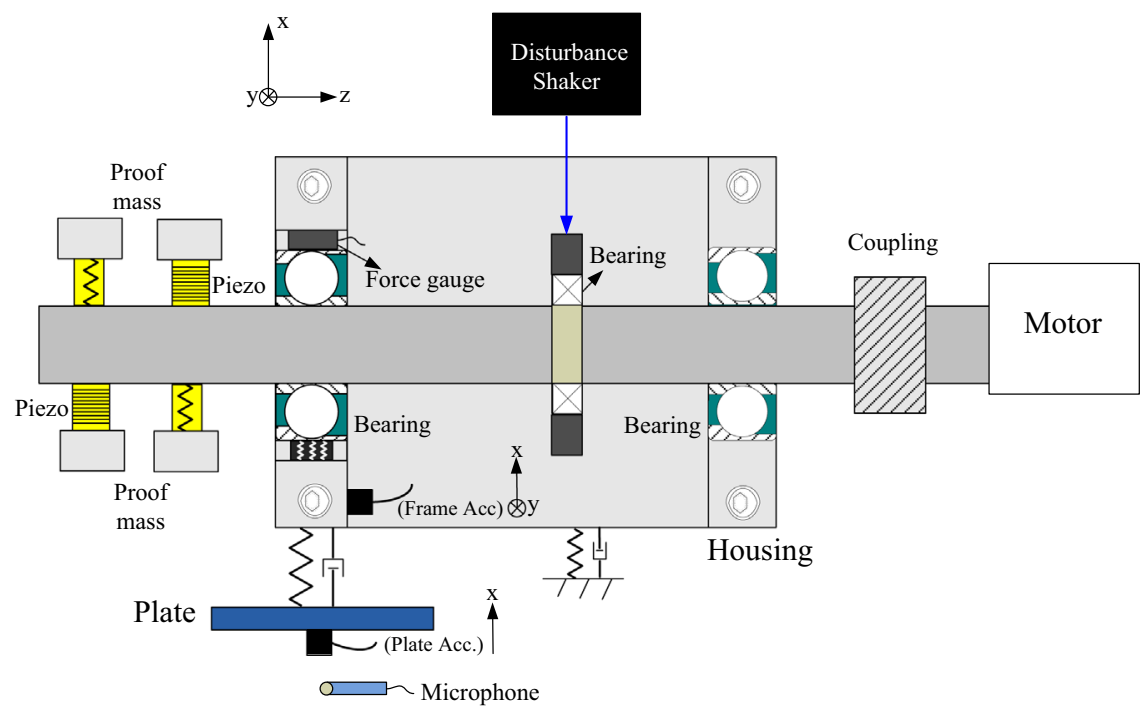

Fig. 5. A cross-sectional view of the experimental set-up with all measurements.

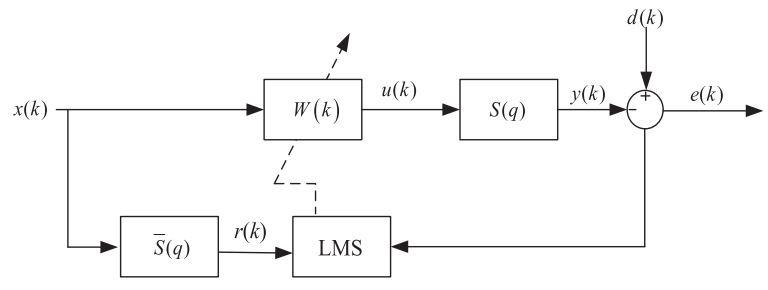

Fig. 6. The block diagram of the FxLMS algorithm.

\section{FxLMS adaptive controller}

In this section, the FxLMS control algorithm is briefly reviewed. Its block diagram is shown in Fig. 6. The core of the control scheme is an adaptive digital filter, which is usually implemented by a finite impulse response filter (FIR), denoted as $W(k)$. The coefficients of $W(k)$ are updated so that the mean square error (MSE) of a chosen error signal $e(k), E\left[e^{2}(k)\right]$ is minimized. This is usually done by using the steepest decent method. Using the instant squared error signal $e^{2}(k)$ to estimate the MSE, the well-known least mean square (LMS) algorithm for updating the coefficients of $W(k)$ is derived:

$$
W(k+1)=\beta W(k)+\mu r(k) e(k)
$$

where $\mu$ is the convergence coefficient, $\beta$ is the power constraint with $0<\beta \leq 1$, e( $k)$ is the instantaneous error, i.e., the difference between the disturbance signal $d(k)$ and the secondary plant $(S(q))$ output $y(k)$, and $r(k)$ is the filtered reference signal which is the convolution of the reference $x(k)$ and the estimated secondary plant $\bar{S}(q)$. Generally, the parameter $\mu$ should be as large as possible without compromising the stability of the system. A suitable value of control effort constraint $\beta$ should be introduced to prevent weight overflow and limit the output power to avoid nonlinear distortion [2]. If the parameter $\beta$ is set to a value other than 1, the FxLMS algorithm becomes the leaky FxLMS algorithm.

Depending on the availability of the reference signal, the FxLMS algorithm can be used to control both broadband and narrowband disturbances. Considering the rotating machinery application, this study focuses on suppressing tonal disturbances and thereby the periodic FxLMS algorithm is implemented and a sinusoidal signal of the same frequency as the disturbance is used as the reference signal. In such a case, the algorithm becomes an adaptive notch filter [30-32]. If the aim is to suppress multiple tones at the fundamental frequency and several harmonics, the reference signal can be composed by a rectangular wave with a period equal to the inverse of the fundamental frequency of the disturbance or simply the sum of all the considered sinusoids [33]. Since two PBRIAs are used in the experiments, the MIMO form of the FxLMS algorithm is investigated. Here, the secondary plants are constructed between the driving signals to the two PBRIAs and the outputs of the two accelerometers measuring the horizontal plate vibrations and the vertical frame vibrations. As mentioned earlier, the chosen error sensors are mounted on the fixed parts of the test bed whereas the piezoelectric actuators are rotating together with the shaft. This means that the constructed secondary plants are continuously changing with respect to the shaft angular position, implying that the secondary plants are time varying.

In order to model the time varying secondary plants, a linear interpolation method is proposed. The idea is to measure the secondary plants at several points in one revolution and approximate the plant variation between those positions by 


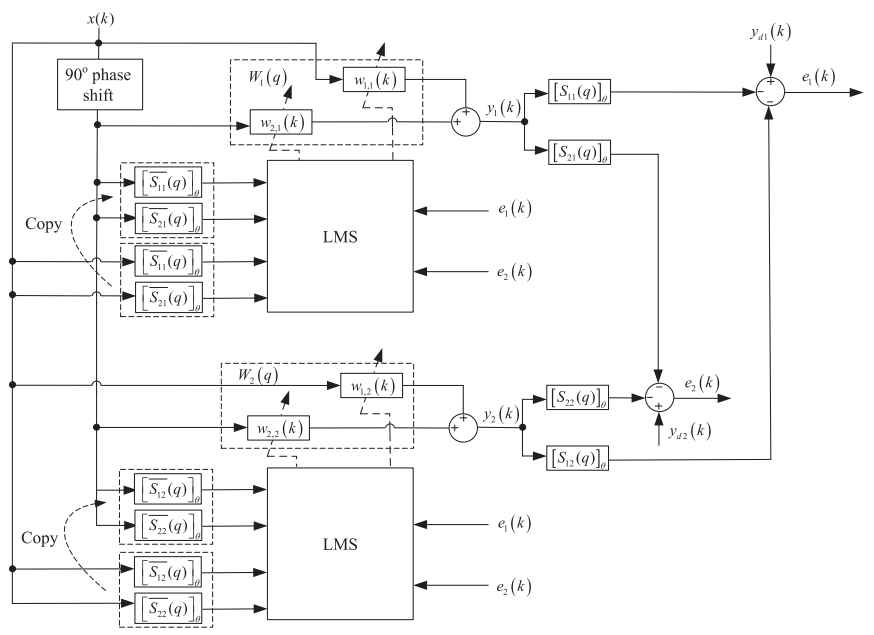

Fig. 7. The flowchart of the MIMO form of the FxLMS algorithm utilising a pair of orthogonal harmonic reference signals.

linearly interpolating between two adjacent pre-identified plants. Note that for IIR filters, interpolation between models is not so easy and it would thus be easier to interpolate on the outputs of these filters. If the secondary plants are modelled by FIR filters however, the interpolation can be made directly on the coefficients of the FIR filters, which is equivalent to an interpolation on the outputs, and simplifies the problem. In practical applications, since the FxLMS algorithm is fairly robust against plant modelling errors [34], it could be expected that the algorithm remains stable as long as the number of the seed plant is large enough such that the phase difference between the plant and the plant model does not exceed $90^{\circ}$.

Finally, the flowchart of the employed MIMO form of the periodic FxLMS algorithm is depicted in Fig. 7, where various symbols have the following meanings: $x(k)$ represents the reference signal which is presumed to be a sinewave and after undergoing a $90^{\circ}$ phase shift, giving the other reference signal $x^{\prime}(k) ; W_{1}(q)$ and $W_{2}(q)$ are the two adaptive FIR filters, each of which is composed by two filter coefficients $w_{i, j}(q), i, j \in[1,2] ; y_{1}(n)$ and $y_{2}(n)$ are the driving signals for the two PBRIAs; $e_{1}(n)$ and $e_{2}(n)$ are the error signals measured by the error sensors; $y_{d 1}(n)$ and $y_{d 2}(n)$ are the disturbance sources measured by the error sensors; $\left[S_{11}(q)\right]_{\theta}$ and $\left[S_{21}(q)\right]_{\theta}$ are the secondary paths from $y_{1}(n)$ to the two error sensors at $\theta$ degrees; $\left[S_{12}(q)\right]_{\theta}$ and $\left[S_{22}(q)\right]_{\theta}$ are the secondary paths from $y_{2}(n)$ to the two error sensors; $\left[\overline{S_{i, j}}(q)\right]_{\theta}$ is the estimate of the secondary plant for $\left[S_{i, j}(q)\right]_{\theta}, i, j \in[1,2]$. The cost function for updating the controller coefficients is the sum of the two squared error signals.

\section{Experimental validation}

The purpose of the experiments in this section is to examine the performance of actively controlled PBRIAs in the test bed presented in Section 2. Prior to describing the results obtained, some system parameters are defined first. During the experimental study a dSpace DS 1006 system has been used both for data acquisition and for control purposes. All the signals fed into the control board or originated from the control board are filtered by a low pass filter with a $1 \mathrm{kHz}$ cut-off frequency. The FxLMS controller is updated at a sampling frequency of $20 \mathrm{kHz}$, but the measured data are recorded at frequency of $2.5 \mathrm{kHz}$ in order to reduce the memory usage for storing of the data. An encoder with a resolution of 1024 pulses per revolution is utilized to measure the rotational position of the shaft, the signals from which are then processed by the dSpace control broad 3001 to calculate the instant angular position. As described earlier, the horizontal acceleration of the plate and the vertical acceleration of the frame are taken as the error signals. The disturbance is provided by the electrodynamic shaker, which is driven by a sinusoidal signal at $371 \mathrm{~Hz}$ throughout the study. This frequency corresponds to one of the resonances of the sound radiating plate; thereby the response of the plate vibrations is much larger than the vertical frame acceleration. Thus, the cost function for the employed FxLMS algorithm is dominated by the plate vibrations. As such, only one accelerometer placed on the plate is in principle enough for the control purpose. The other error sensor is chosen to avoid a possible enhancement of the frame vibrations in the vertical direction due to the control action. The whole control scheme is implemented in the Matlab Simulink environment and then downloaded to the processor unit of a dSpace 1006 system. The sine wave sent to the disturbance shaker is directly taken as the reference signal for the FxLMS controller in the Simulink model. In such a case, a perfect correlation between the reference and the disturbance is assumed. In reallife applications, a tachometer signal, which provides information concerning the disturbance frequency, is always taken as the reference signal. This signal can be acquired for example from an optical sensor measuring the rotational motor speed. In order to assure an adequate level of coherence between the reference and disturbance signals, the measured pulse train can be fed into a frequency estimator to estimate the instant rotating speed [22]. 


\subsection{Secondary plant modelling and identification}

The pre-identified secondary plants are modelled by FIR filters, which can be estimated off-line with an LMS adaptive algorithm [2]. The process consists of exciting the secondary path with a sine wave and simultaneously providing the same signal as the reference to a conventional LMS algorithm. After the convergence of the algorithm, the controller coefficients will then resemble the secondary path impulse response. Since only a single frequency is considered, an FIR filter with only 2 coefficients is sufficient. By repeating this at multiple frequencies, the FIR filter coefficients for the secondary plants at all relevant frequencies can be assembled in a lookup table as a function of the disturbance frequency. An alternative is to measure the FRFs of the secondary paths in a broad frequency band, from which the FIR coefficients at one frequency can also be derived.

As mentioned above, the constructed secondary plants are angular position dependent and an interpolation scheme is needed. Here, each secondary plant is identified at 32 angular positions in one revolution with an interval of $11.25^{\circ}$. The $0^{\circ}$ position is defined when piezoelectric actuator of PBRIA 1, as shown in Fig. 4, is parallel to the disturbance. Fig. 8(a) and (b) show the amplitude and phase of the secondary-plant FRFs between the voltages to the piezoelectric actuators and the

(a)

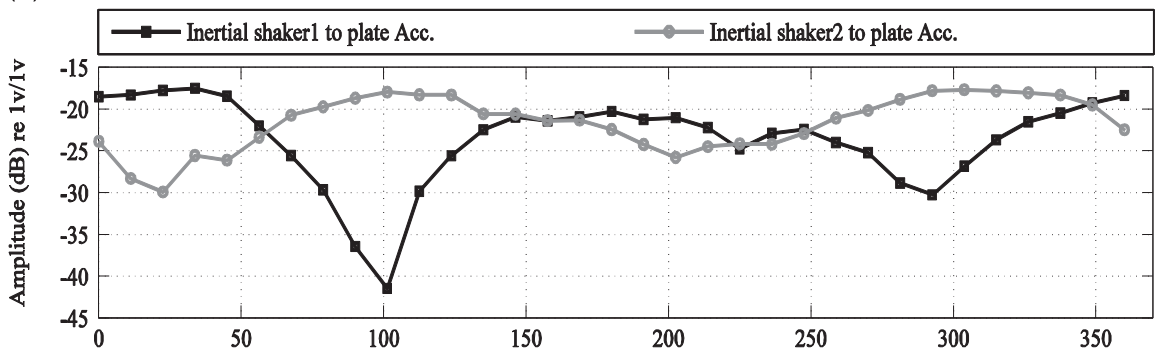

(b)

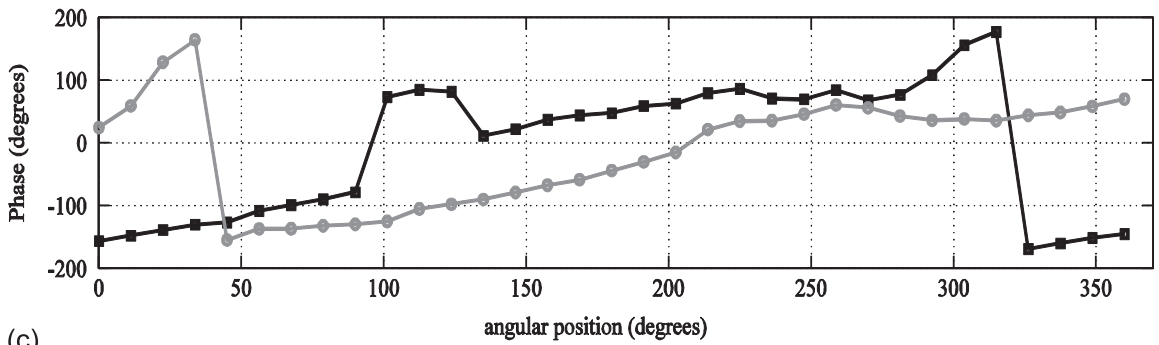

(c)

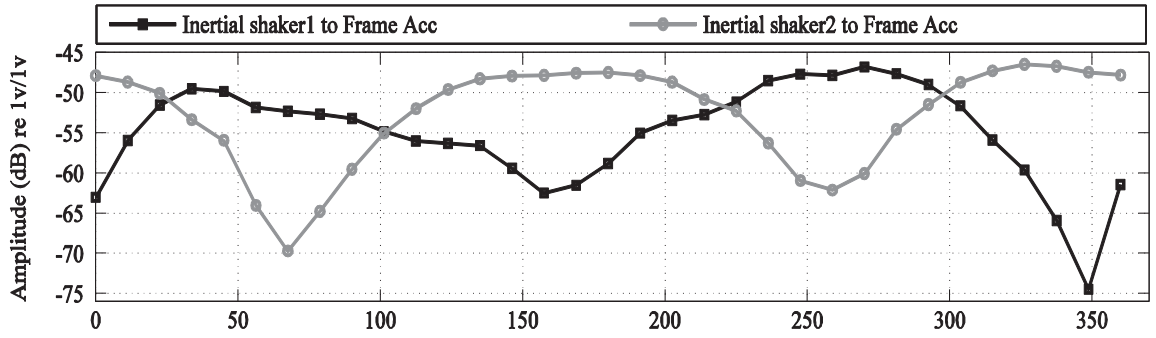

(d)

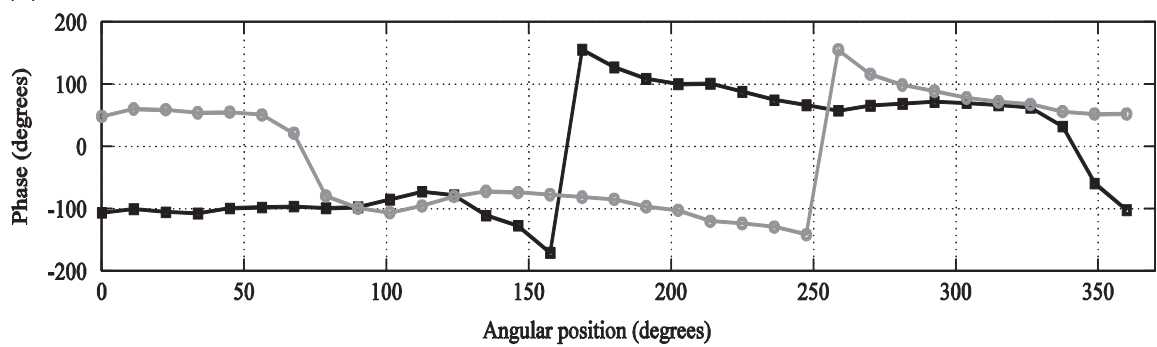

Fig. 8. Frequency response functions of the secondary plants at the 32 angular positions: (a) and (b), the amplitude and the phase of the FRFs between PBRIAs 1 and 2 to the horizontal plate vibration; (c) and (d), the amplitude and the phase of the FRFs between PBRIAs 1 and 2 to the vertical frame vibration. 
measured plate vibrations at $371 \mathrm{~Hz}$, while Fig. 8(c) and (d) present the amplitude and phase of the other two secondary plants between the voltages to the piezoelectric actuators and the measured vertical frame vibrations. As can be seen, the four plants vary with respect to the angular positions, where the crests and troughs of the curves are located at these positions when the actuation direction of the PBRIA is parallel and perpendicular to the direction of the sensors.

In the following sections, the performance of the developed controller is demonstrated in two conditions. Firstly, the shaft is not rotating. The experiments are concerned with the effect of the controller to suppress the response of different parts of the test bed. In the second step, the shaft is rotating. The focus is placed on examining the control effectiveness over a selected range of operating speeds and the number of the pre-identified secondary plants.

\subsection{Non-rotating tests}

The potential of the proposed actively controlled PBRIAs is first illustrated when the shaft is fixed at $0^{\circ}$ position. The convergence rate $\mu$ is set to 0.3 which is about half of the critical value after which the system becomes unstable. The leakage factor $\beta$ is set to 0 which means no power constraint is considered in this case. In Fig. 9(a) and (b), the time histories of the two error signals are presented, which are the horizontal plate acceleration and the vertical frame acceleration respectively. First, up to $t=5 \mathrm{~s}$, the controller is inactivated and later on it is activated. It can be seen that after convergence the accelerations have decreased dramatically, with reductions of $45 \mathrm{~dB}$ and $31 \mathrm{~dB}$ respectively. The convergence period takes approximately $0.1 \mathrm{~s}$ and $20 \mathrm{~s}$, and thus differs strongly for both. For the MIMO FxLMS control algorithm, the speed of convergence is mainly determined by two factors, which are the initial excitation levels and the spatial eigenvalues of the secondary plants matrix [3]. Here, the chosen frequency of $371 \mathrm{~Hz}$ coincides with one of plate resonances so that the response picked up by the accelerometer on the plate is about 10 times larger than the one measuring the vertical vibration level of the frame at this frequency, which already partially explains why the convergence for the plate accelerations is much faster. Besides this, the spatial eigenvalue corresponding to the plate vibrations is calculated to be much larger than that of the frame vibrations. The two eigenvalues can be derived from the matrix $M=\bar{S}^{H} \bar{S}$, where $\bar{S}$ is composed by

$$
\bar{S}=\left[\begin{array}{ll}
{\left[\overline{S_{1,1}}(q)\right]_{0}} & {\left[\overline{S_{2,1}}(q)\right]_{0}} \\
{\left[\overline{S_{1,2}}(q)\right]_{0}} & {\left[\overline{S_{2,2}}(q)\right]_{0}}
\end{array}\right]
$$

In Eq. (2), $\left[\bar{S}_{i, j}\right]_{0}, i, j \in[1,2]$ is a complex quantity representing the frequency response function of the corresponding model of the secondary plant at $0^{\circ}$ position in Fig. 8.

Therefore, it is reasonable to obtain the difference of the convergence speed. In order to equalize the convergence speeds, the cost function or the position of sensors could be altered. This is however not necessary in the present study, since the

(a)

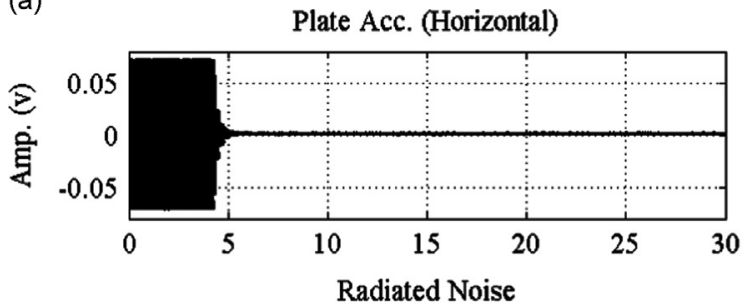

(c)

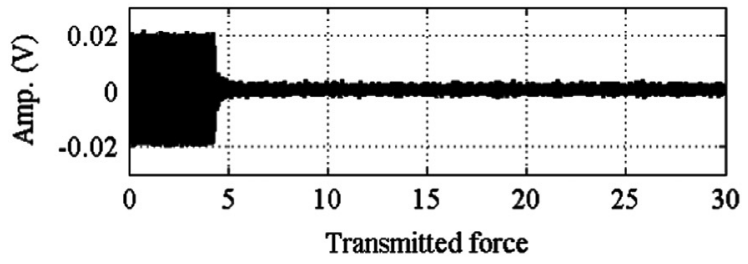

(e)

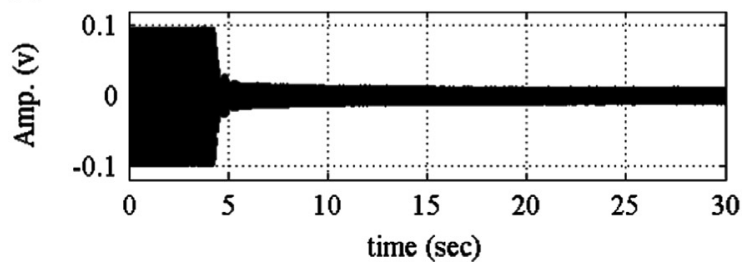

(b)

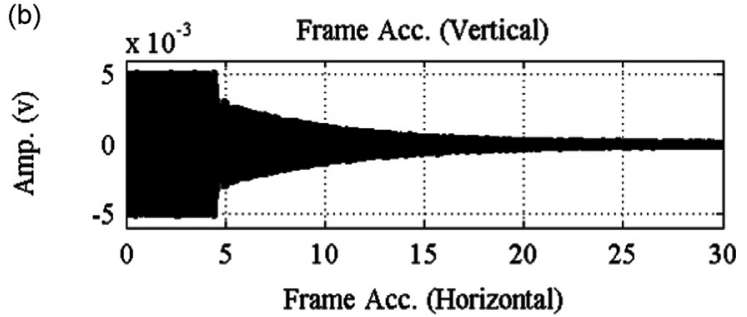

(d)

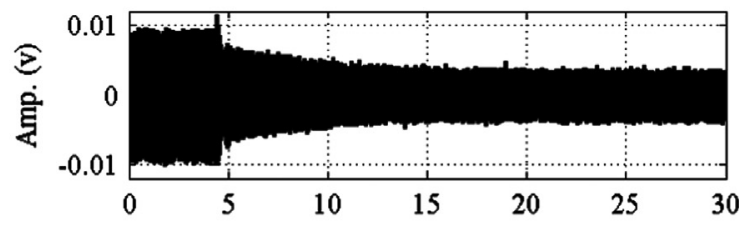

Fig. 9. The control effect on (a) plate vibrations, (b) vertical frame vibrations, (c) radiated noise, (d) horizontal frame vibrations and (e) transmitted force. 
interest is primarily in reducing the radiated noise. The frame vibrations are included in the sum of the squared errors only to ensure that no excessive vibration amplifications occur at the frame.

Fig. 9(c)-(e) plot the radiated noise, horizontal frame acceleration and the transmitted force respectively. From these, it can be seen that a reduction of the error signals also leads to a reduction in the audible noise, the transmitted force and the frame vibrations. A global response reduction is obtained, primarily because the response of the measured points is dominated by the plate mode. The reduction of the frame vibrations in the horizontal direction as well as the transmitted force is less pronounced since those parameters were not included in the error signal. As the FxLMS algorithm only guarantees reductions at the error sensors, the location of the PBRIAs should also be considered carefully, and they should ideally be placed as close as possible to the disturbance such that they excite the structure in the same manner as the disturbance. Nevertheless, the results confirm that for a periodic disturbance, the reductions of the error signals are significant.

\subsection{Rotating tests}

In this subsection, experiments with the proposed PBRIAs are performed when the shaft is rotating at 60,120 and $180 \mathrm{rpm}$ respectively in order to investigate the influence of the rotating speed on the performance. In the first set of experiments, a non-adaptive feedforward controller is developed to serve as a benchmark case. This controller is similar to the proposed MIMO FxLMS controller, which also consists of two 2 order FIR control filters. The difference is that the controller filter coefficients are not updated by the LMS scheme during normal operation, but are predefined. First, during a calibration phase, the MIMO form FxLMS algorithm is used to identify the optimal FIR filter control coefficients at the 32 angular positions, where the secondary plants were already identified, with the shaft not rotating and the disturbance kept at the same level. In the non-adaptive controller scheme those controller coefficients are the basis to derive the instant angular dependent control coefficients corresponding to the current angle using a linear interpolation scheme. The motivation for doing so is that by using the predefined optimal control coefficients, the influence of the convergence speed can be eliminated.

Fig. 10(a) plots the time histories of the plate vibration at the rotating speed of $60 \mathrm{rpm}, 120 \mathrm{rpm}$ and $180 \mathrm{rpm}$ (from top to bottom), first with controller deactivated, then activated, and then again deactivated. A substantial reduction is thus obtained for each case when the controller is switched on. However the residue level slightly increases with an increase of the rotating speed, which indicates that the reduction performance might further degrade as the shaft operates faster. In order to further understand these results, the achieved reductions are plotted as a function of the shaft rotational position. To do so, the time domain signals of the first two segments (deactivated and activated) are synchronized with the rotating

(a)
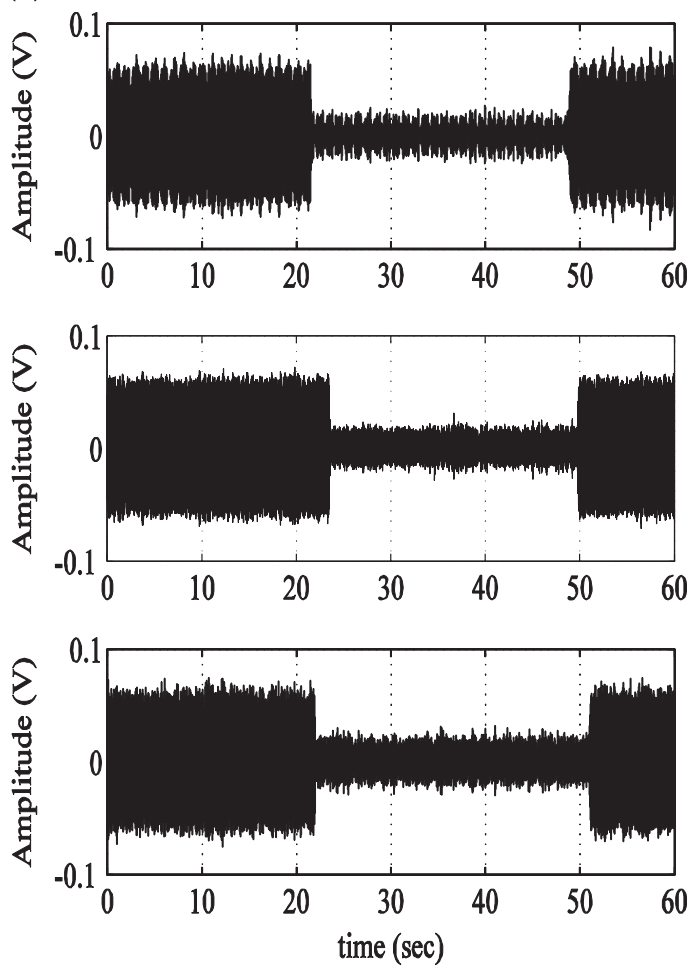

(b)
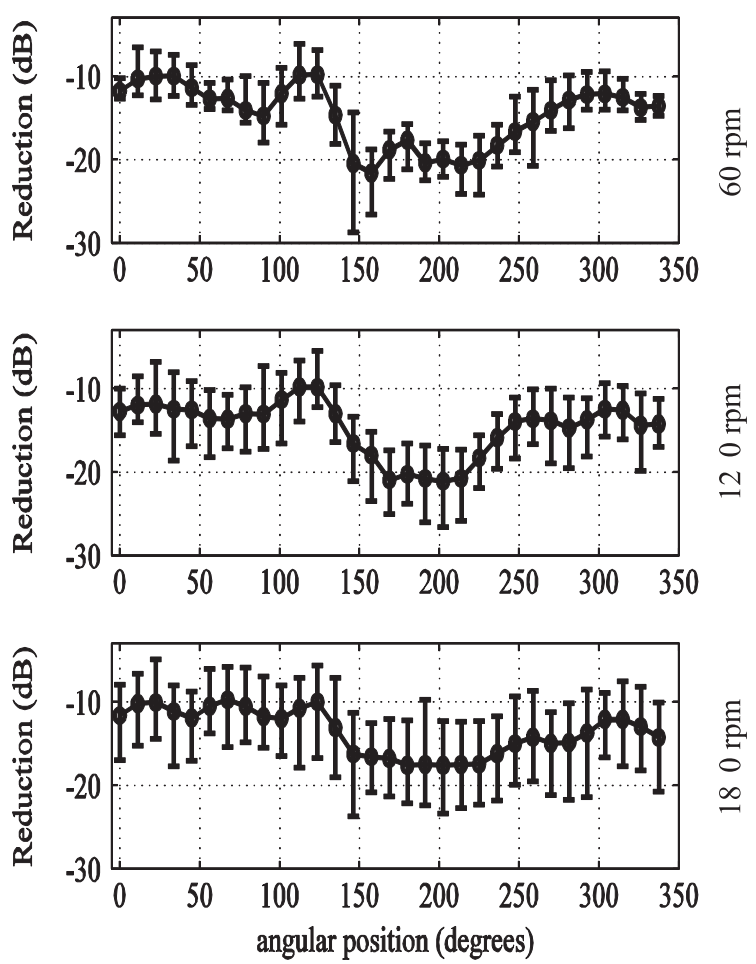

Fig. 10. (a) The effect of the pre-learned controller on the plate acceleration when the shaft rotates at, from top to bottom, $60 \mathrm{rpm}, 120 \mathrm{rpm}$ and $180 \mathrm{rpm}$ in the time domain; (b) The resultant reductions in the angular domain. 
speed signal measured by the encoder, and the reductions are calculated in an interval of $10^{\circ}$. The resultant reductions are shown in the angular domain in Fig. 10(b), where the average reductions are represented by the dot-line and the variations during the different revolutions are indicated as the error bars. As shown, the trend of the averaged reductions seems to be independent of the rotating speed, while the variation level does increase with an increase of the speed. Furthermore, in each case, despite the trend being the same, the averaged reductions do vary with the angular position (Fig. 10(b)), and more importantly the reductions are much smaller than those obtained previously for the non-rotating shaft. The above phenomenon can be caused by the following aspects: (1) the non-perfect estimation of the controller coefficients and the secondary plants; (2) nonlinearities of the secondary plants, which means the secondary plants might vary in terms of the rotation speed; (3) the low damping characteristic of the system which means the control actuation does not decay quickly enough from one angular position to another.

Fig. 11(a) shows the frequency spectra of the first two segments of the time domain signals, and Fig. 11(b) shows the reductions at individual spectral lines (the disturbance frequency itself and three pairs of rotation speed harmonics). It can be seen that a reduction of approximately $19 \mathrm{~dB}$ is obtained at the exciting frequency, independent of the rotation speed. However, the reduction at the adjacent harmonics is dependent on the rotation speed. The undesired amplification of more than $5 \mathrm{~dB}$ at the first rotation speed harmonic above the disturbance frequency might be attributed to the fact that the control is only aimed at the zero-order harmonic at $371 \mathrm{~Hz}$.

The control effect on the second error sensor (frame acceleration in the vertical direction) is shown in Fig. 12(a), again for rotating speeds of $60 \mathrm{rpm}, 120 \mathrm{rpm}$ and $180 \mathrm{rpm}$ (from top to bottom). Here, the loss of the control effectiveness is more obvious, and for the $180 \mathrm{rpm}$ case the effect is almost unnoticeable. The results are further examined in the angular domain, which are shown in Fig. 12(b). In the angular domain, the vibrations are even amplified between $75^{\circ}$ and $125^{\circ}$. This is probably because the optimal control coefficients were not identified accurately for this angular range.

Finally, the spectra of the vertical frame vibrations with and without control are shown in Fig. 13(a). The corresponding reductions at the disturbance frequency and three adjacent harmonics are shown in Fig. 13(b). It is clear that the control is most effective at the disturbance frequency.

In the second set of experiments, the controller coefficients are directly updated online using the FxLMS algorithm, as would be done during normal operation. The convergence rate $\mu$ is set to 0.7 and the leakage factor $\beta$ is set to 0.995 . Here, certain power constraint is introduced, which is essential for the current MIMO control case where the plant matrix is nearly ill-conditioned. By doing so, unreasonable values of control effort are prevented, the risk of instability is reduced and the convergence rate of the insignificant errors (frame vibrations) is improved [2].

(a)
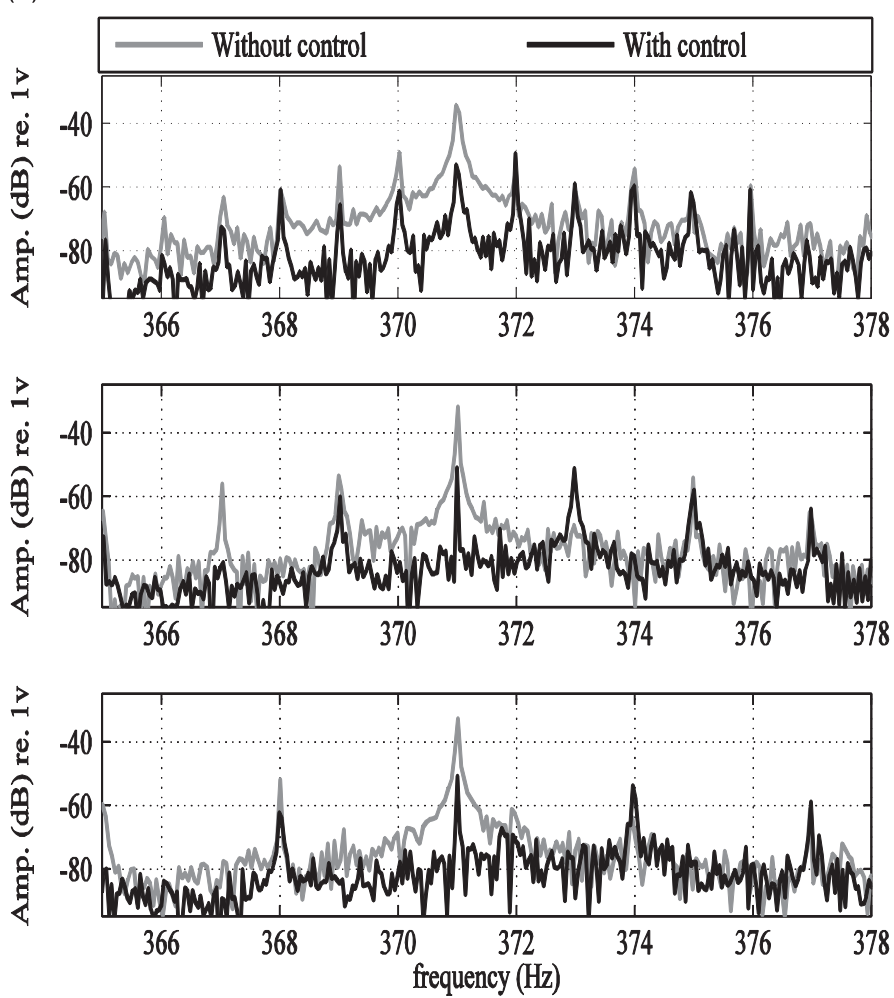

(b)
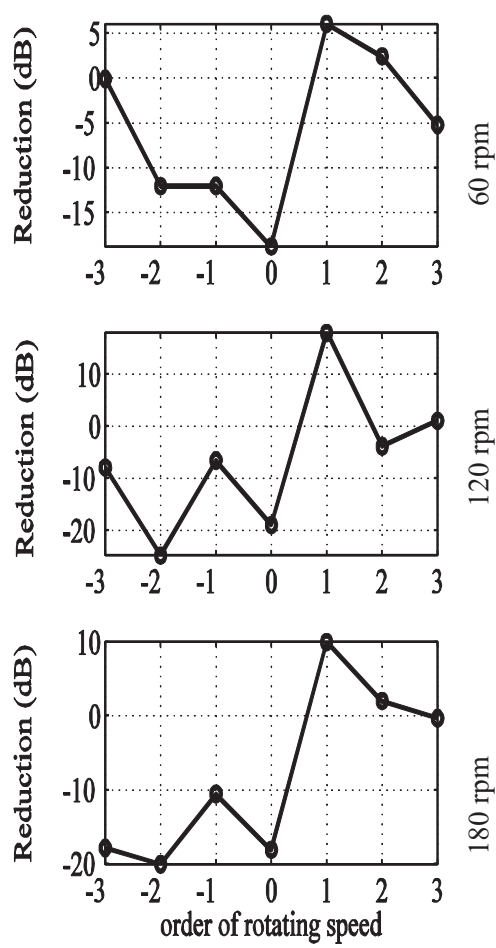

Fig. 11. (a) Comparison of the plate vibrations in the frequency domain, without control and with control when the shaft rotates at, from top to bottom, $60 \mathrm{rpm}, 120 \mathrm{rpm}$ and $180 \mathrm{rpm}$; (b) the resultant reductions at the disturbance frequency itself and three pairs of adjacent harmonics. 
(a)
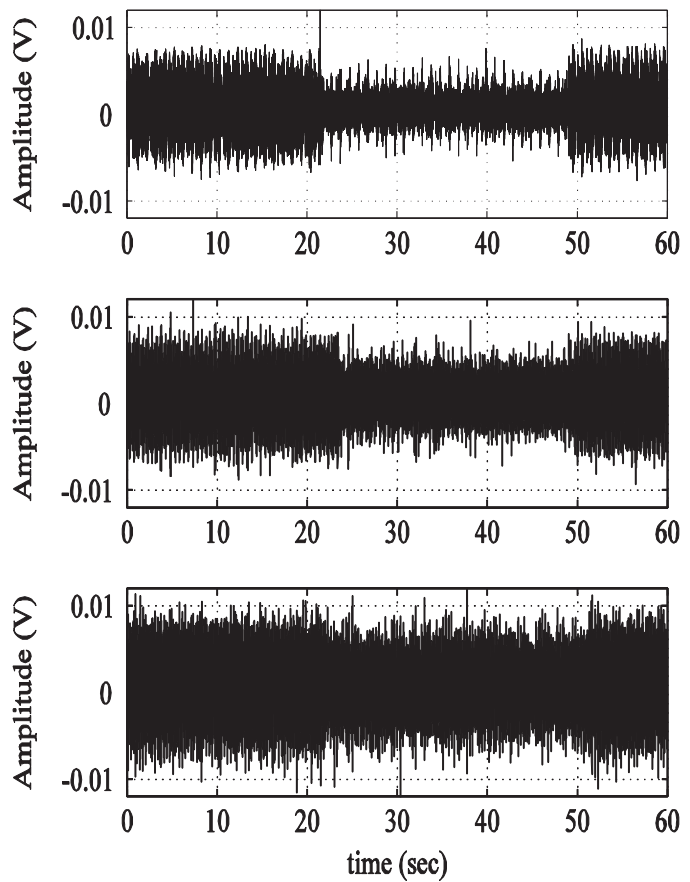

(b)
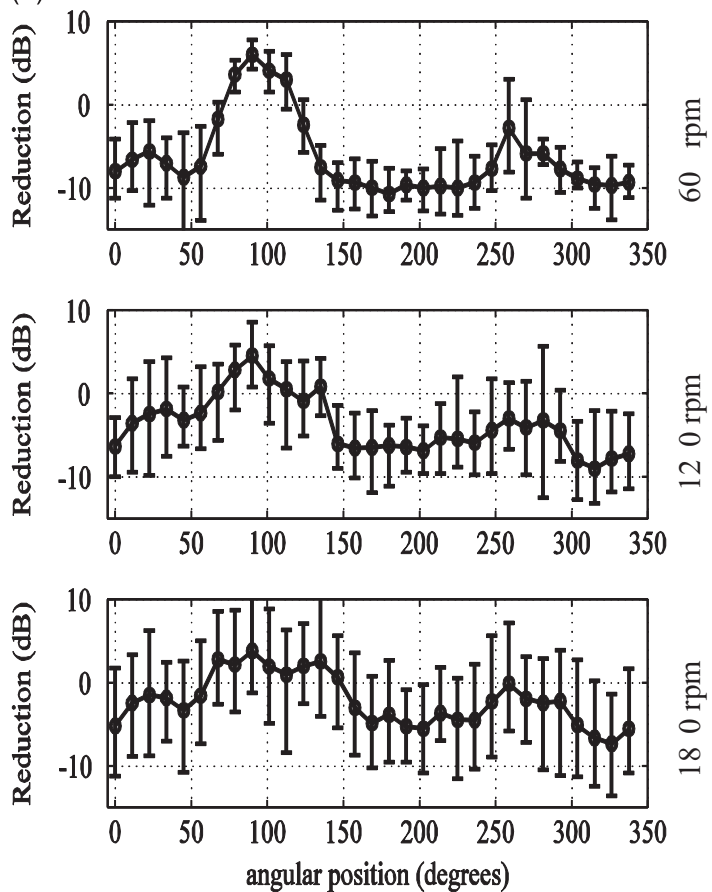

Fig. 12. (a) The effect of the pre-learned controller on the vertical frame acceleration when the shaft rotates at, from top to bottom, 60 rpm, 120 rpm and $180 \mathrm{rpm}$ in the time domain; (b) The resultant reductions in the angular domain.

(a)
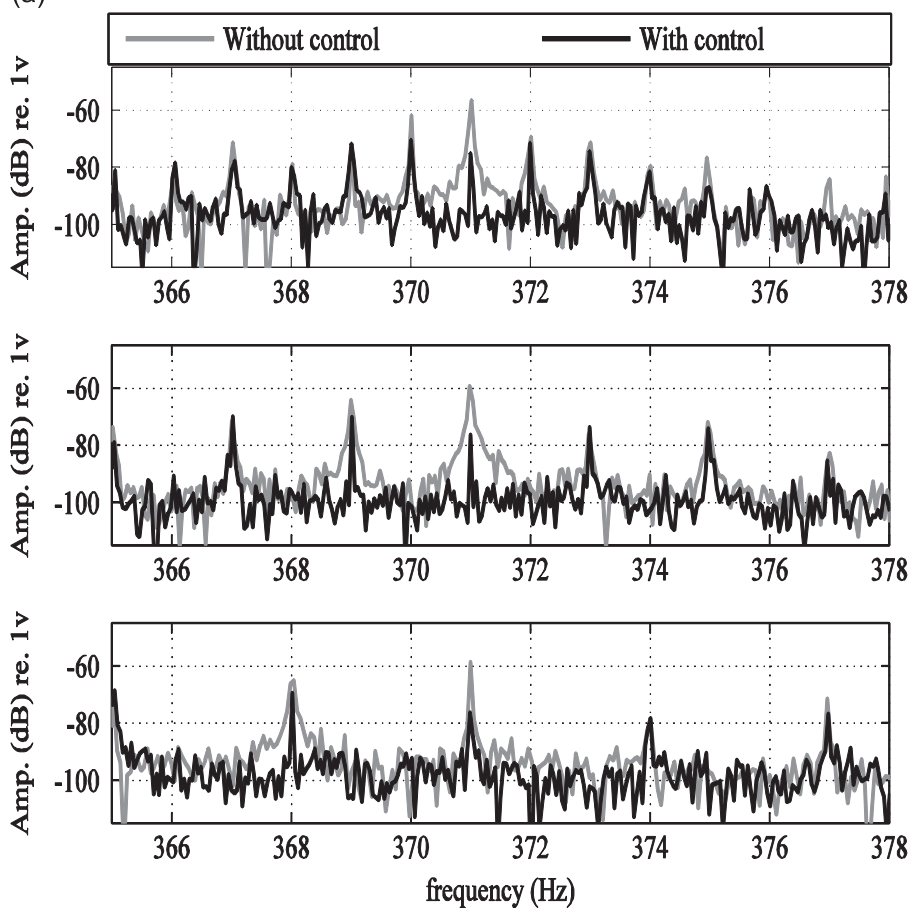

(b)
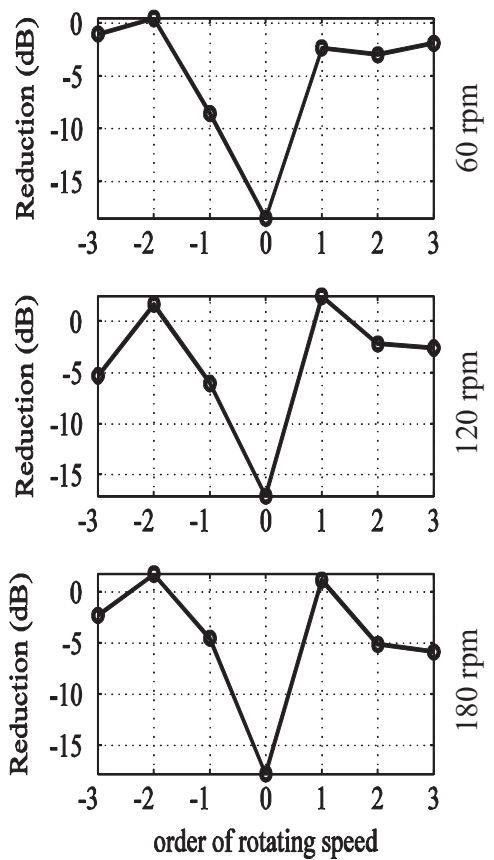

Fig. 13. (a) Comparison of the vertical frame vibrations in the frequency domain, without control and with control when the shaft rotates at, from top to bottom, $60 \mathrm{rpm}, 120 \mathrm{rpm}$ and $180 \mathrm{rpm}$; (b) the resultant reductions at the disturbance frequency itself and three pairs of adjacent harmonics.

Fig. 14(a) shows the measured plate vibrations in the same three running conditions as before, again with the control first deactivated, then activated and then again deactivated. The corresponding angular domain reductions for the three cases are plotted in Fig. 14(b). The loss of the control effect is now more noticeable as the rpm increases. The amplitude spectra of the 
(a)
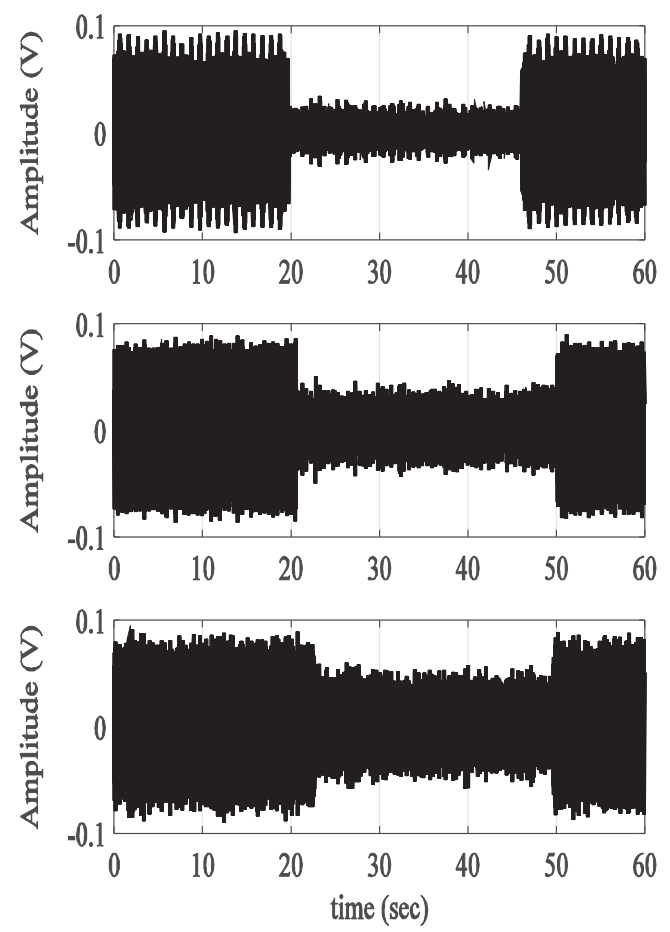

(b)
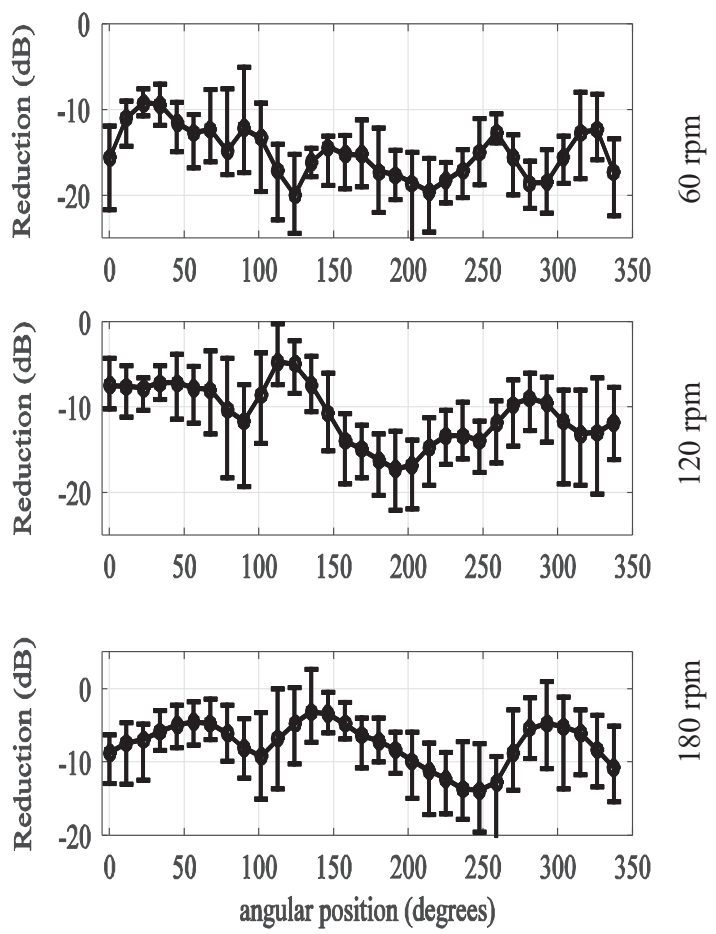

Fig. 14. (a) The effect of FxLMS controller on the plate acceleration when the shaft rotates at, from top to bottom, 60 rpm, 120 rpm and 180 rpm in the time domain; (b) the resultant reductions in the angular domain.

measured plate vibrations with and without control are shown in Fig. 15(a), while Fig. 15(b) shows the reductions at individual spectral lines (the disturbance frequency and adjacent harmonics). Here the achieved reductions at the excitation frequency reduce with increasing rpm. This was not the case with the previous experiments where the non-adaptive controller with pre-defined controller coefficients was used. The main reason for the loss of the performance with respect to the previous experiments is that the faster change on the secondary plants makes it more challenging for the controller to follow and adapt to these changes. Non-perfect estimation of the secondary plants and their nonlinearities may also contribute to the control effectiveness loss.

The control performance in terms of the vertical frame vibrations reduction is also examined, as shown in Figs. 16 and 17. Compared to the plate vibrations, the control effectiveness has now completely disappeared and amplifications are observed for the experiment performed at $180 \mathrm{rpm}$. This is mainly because the convergence speed of the vertical frame vibration is much less than that of the plate vibration such that the initial transient of the controller coefficients are mainly determined by the plate vibration. With the increase of the rotating speed, the performance loss on the plate vibrations, as shown in Figs. 14 and 15, thus leads to the poor control effectiveness on the vertical frame vibrations. Since the emphasis in the study is on the plate vibrations, the behaviour of the vertical frame acceleration is to be expected.

Finally, the influence of the number of pre-identified secondary plants onto the performance of the present control approach is studied. This is done by comparing the achieved reductions for the FxLMS controller with varying numbers of the pre-identified secondary plants: 4 and 32. Experiments have also been carried out with 2, 8, 16 and 64 pre-identified secondary plants, however the achieved reductions are virtually the same if the number of the interpolation plants was larger than 4. It was found that it is only when the number of the predefined secondary plant is 4 that the performance is slightly reduced, and if it is 2 the algorithm is unstable for any rotation speed.

Fig. 18(a) and (b) compare the control effectiveness of the FxLMS controllers with two characteristic numbers (4 and 32) of the pre-identified secondary plants. As can be seen in Fig. 18(a), the control performance in terms of the residue level of the plate vibrations with control looks very similar for the two cases. However, there is a slight improvement of the control effectiveness when using 32 interpolation points at higher rpms. This can be better seen in the angular domain (Fig. 18(b)) between $110^{\circ}$ and $160^{\circ}$. It was found that this is due to a mismatch between the amplitude and the phase of the secondary plants pre-identified with 4 and 32 interpolation points. This mismatch lowers the convergence rate of the 4 point interpolation algorithm and thus slightly decreases the end performance. At lower rpms, the controller has relatively sufficient time to converge such that the difference in terms of the achieved reductions is negligible. However, this is not true at higher rpms since in these cases the FxLMS controller has less time to update the coefficients and thus the control performance loss due to the modelling error is more pronounced. 


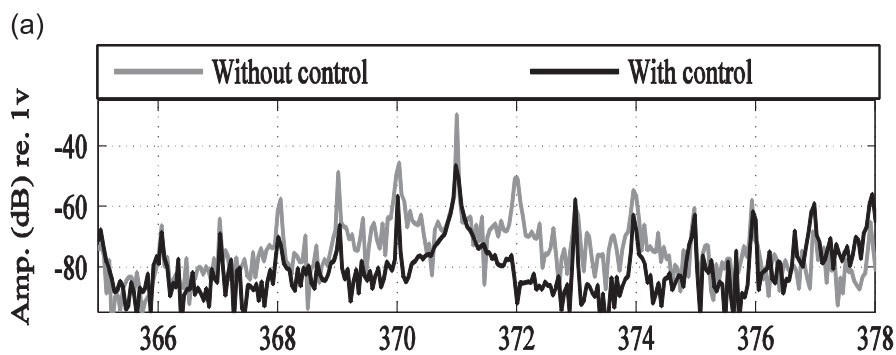

(b)
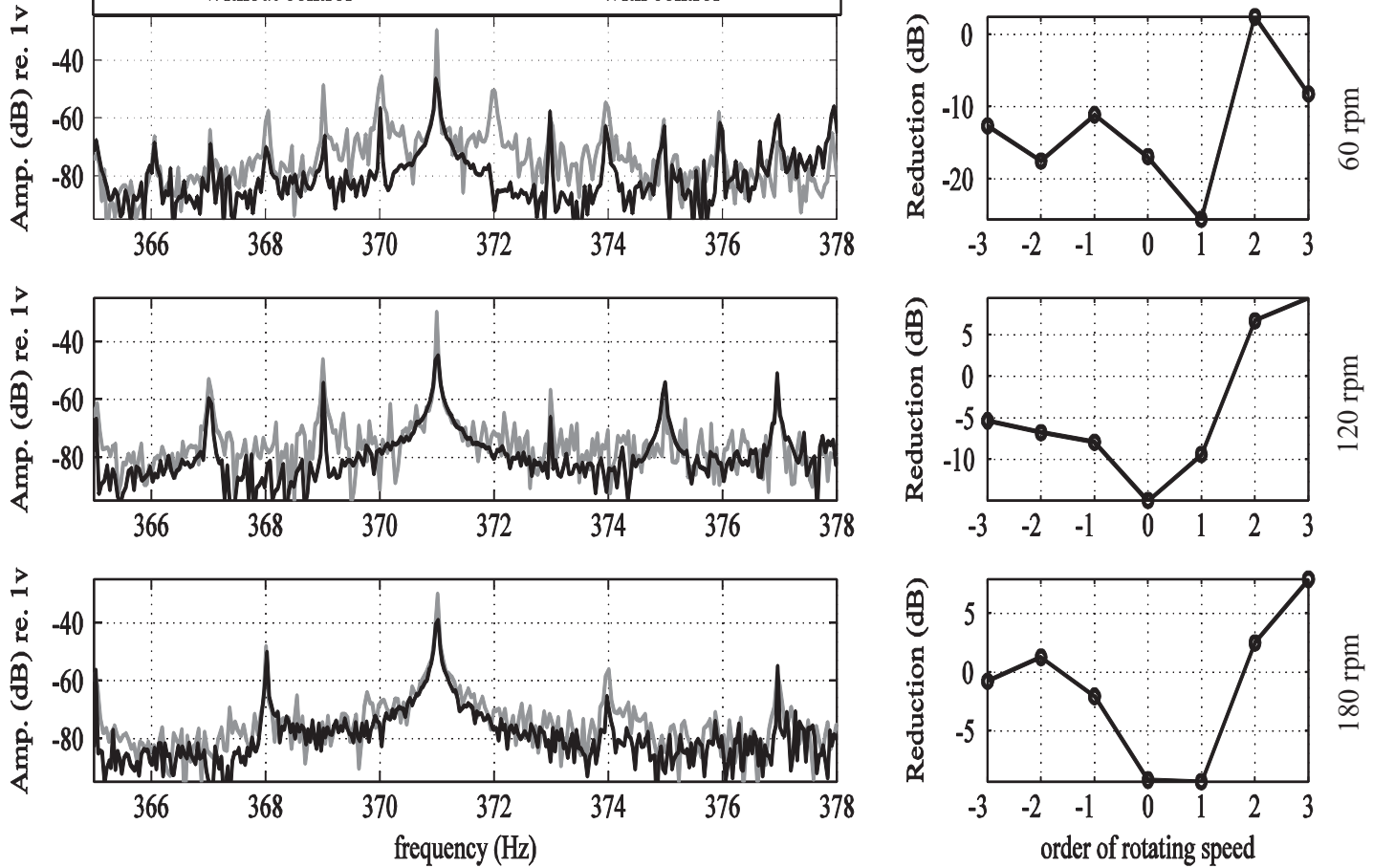

Fig. 15. (a) Comparison of the plate vibration, without control and with control when the shaft rotates at, from top to bottom, $60 \mathrm{rpm}, 120 \mathrm{rpm}$ and $180 \mathrm{rpm}$ in the frequency domain; (b) the resultant reductions at the disturbance frequency itself and three pairs of adjacent harmonics.

(a)
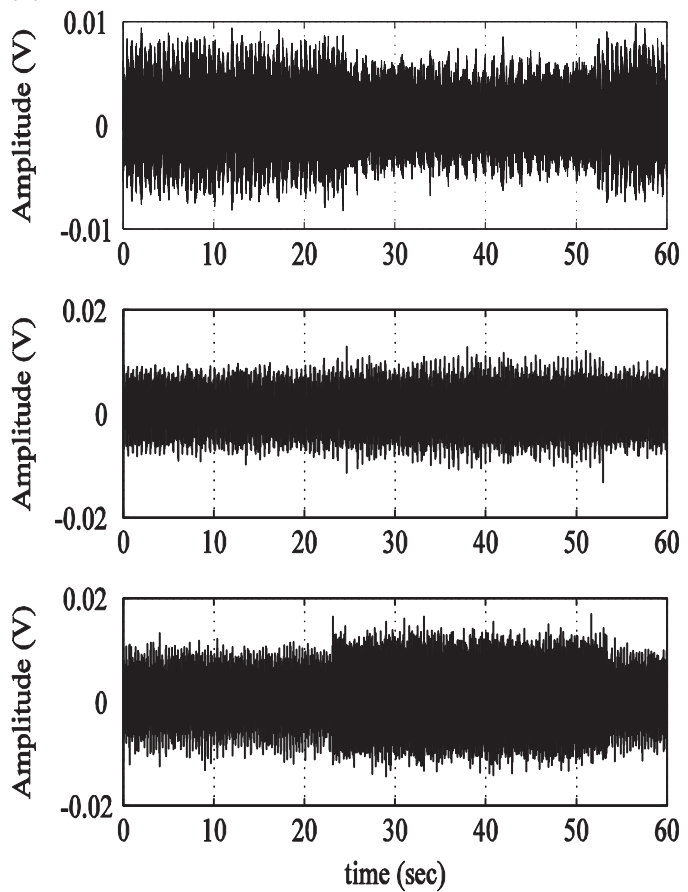

(b)
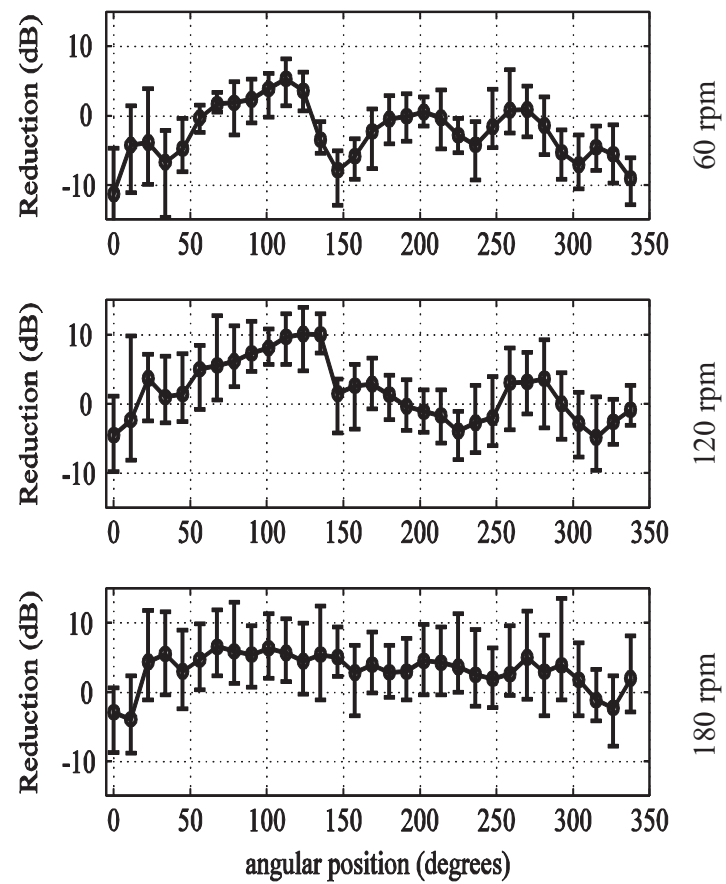

Fig. 16. (a) The effect of FxLMS controller on the vertical frame vibrations when the shaft rotates at, from top to bottom, $60 \mathrm{rpm}, 120 \mathrm{rpm}$ and $180 \mathrm{rpm}$ in the time domain; (b) The resultant reductions in the angular domain. 
(a)
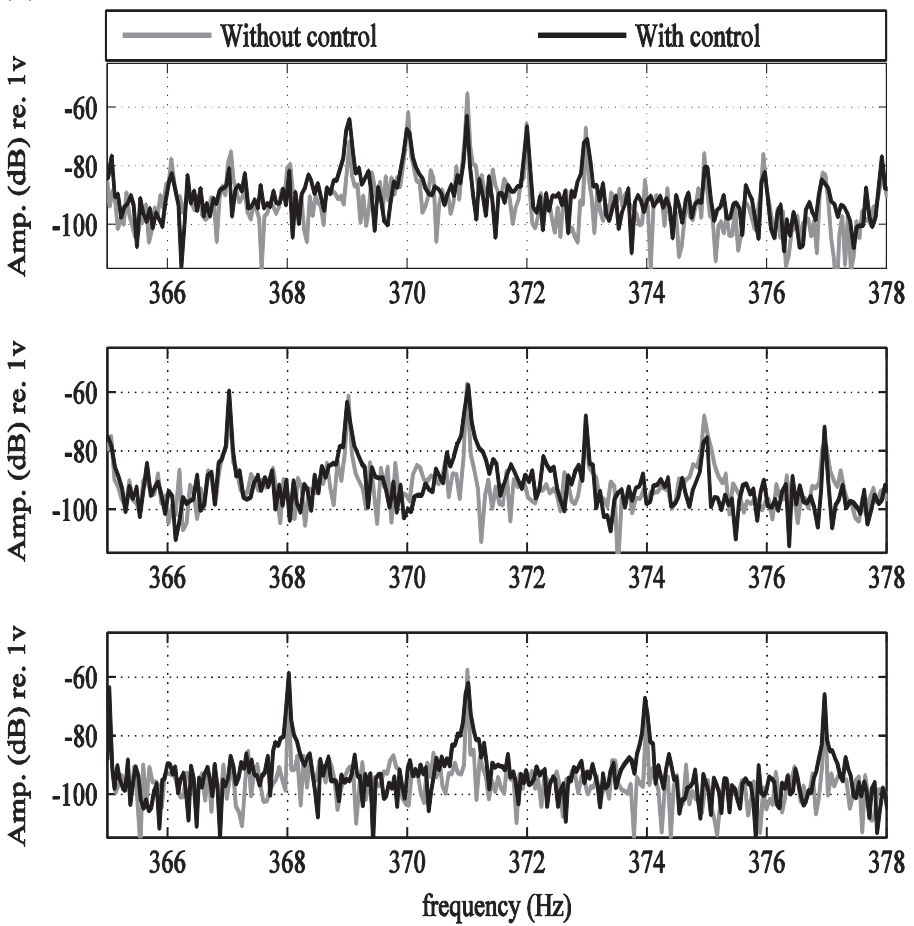

(b)
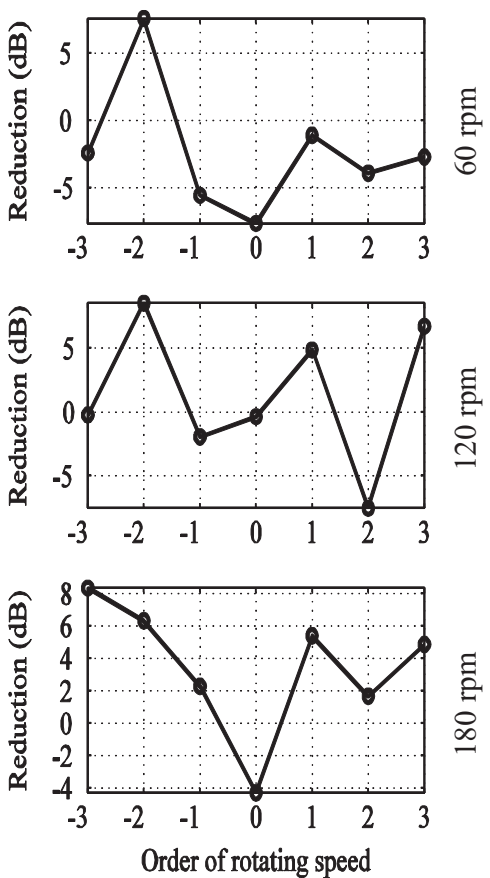

Fig. 17. (a) Comparison of the vertical frame vibrations in the frequency domain, without control and with control when the shaft rotates at, from top to bottom, $60 \mathrm{rpm}, 120 \mathrm{rpm}$ and $180 \mathrm{rpm}$; (b) the resultant reductions at the disturbance frequency itself and three pairs of adjacent harmonics.

(a)
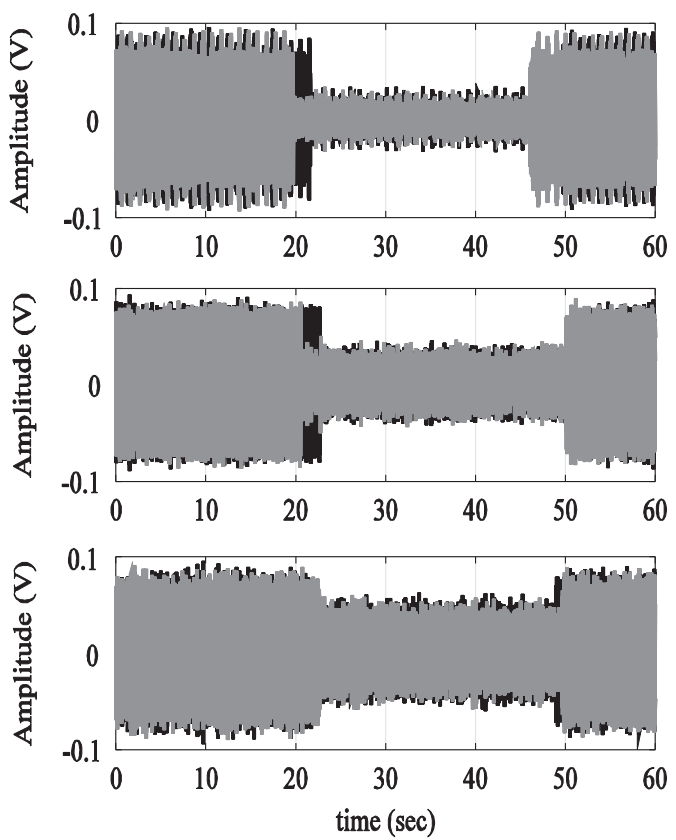

(b)
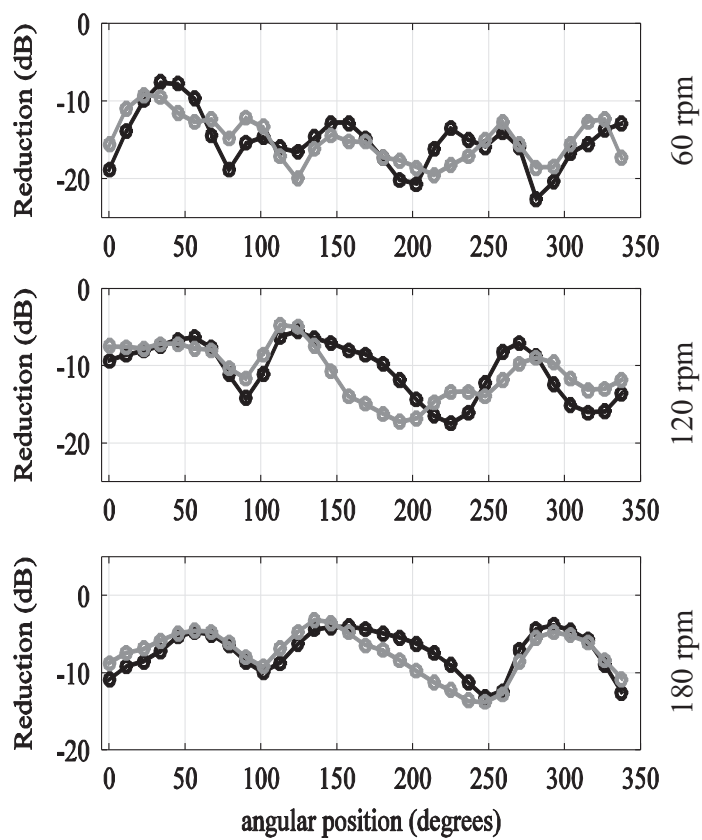

32 interpolation points

Fig. 18. (a) Comparison of the effect of the FxLMS controller with different interpolation points on the plate vibrations in the time domain when the shaft rotates at, from top to bottom, $60 \mathrm{rpm}, 120 \mathrm{rpm}$ and $180 \mathrm{rpm}$; (b) Comparison of the resultant average reductions during different revolutions in the angular domain. 


\section{Conclusions}

This paper discusses a novel control concept for suppressing rotating machinery radiating noise, which is to use PBRIAs that rotate together with the machinery to actively control the effects of disturbance forces transmitted to the structure housing. A MIMO form of the FxLMS algorithm is applied to control the plate vibrations and the frame vibrations. In order to account for the time varying effects of the rotating PBRIAs, a linear interpolation scheme is proposed to model the time varying secondary plants. The design and control approach have been validated on an experimental test bed. It has been shown that more than $7 \mathrm{~dB}$ reductions in the plate vibrations can be achieved when the shaft rotates below 180 rpm, with the resulting reduction of acoustic noise in the same order of magnitude. These results demonstrate the technical feasibility of using the considered PBRIAs for suppressing structure borne noise of rotating machinery for applications with a low running speed. Once the speed becomes high however, the controller can have difficulties following and adapting fast enough. Therefore, future studies will aim at improving the performance of the active vibration control approach for higher rotating speeds, as well as gaining a better understanding of the limitations of the followed approach.

\section{Acknowledgements}

The Research Fund KU Leuven is gratefully acknowledged for its support. The China Scholarship Council is also gratefully acknowledged. The IWT Flanders within the OPTIWIND project (GA: IWT/120029) is gratefully acknowledged for its support. The European Commission is gratefully acknowledged for their support of the ANTARES research project (GA 606817).

\section{References}

[1] P.A. Nelson, S.J. Elliott, Active Control of Sound, Academic Press, New York, 1991.

[2] S.M. Kuo, D.R. Morgan, Active Noise Control Systems: Algorithms and DSP Implementation, Wiley, New York, 1996.

[3] S.J. Elliott, C.C. Boucher, P.A. Nelson, The behaviour of a multiple channel active control system, IEEE Transactions on Signal Processing 40 (5) (1992) $1041-1052$.

[4] S.J. Elliott, P.A. Nelson, I.M. Stothers, C.C. Boucher, Inflight experiments on the active control of propeller-induced cabin noise, Journal of Sound and Vibration 140 (1990) 219-238.

[5] W. Dehandschutter, The Reduction of Structure-Borne Noise by Active Control of Vibration PhD thesis, KU Leuven University, Leuven, Belgium, 1997.

[6] G. Pinte, Active Control of Repetitive Impact Noise PhD thesis, KU Leuven University, Leuven, Belgium, 2007.

[7] J.P. Den Hartog, Mechanical Vibrations, McGraw-Hill Book Co., New York, 1934.

[8] J.B. Hunt, Dynamic Vibration Absorbers, Mechanical Engineering Publications Ltd, London, 1979.

[9] D.J. Inman, Engineering Vibration, Prentice-Hall, New York, 1994.

[10] N. Alujevic, I. Tomac, P. Gardonio, Tunable vibration absorber using acceleration and displacement feedback, Journal of Sound and Vibration 331 (12) (2012) 2713-2728.

[11] C. Paulitsch, P. Gardonio, S.J. Elliott, P. Sas, R. Boonen, Design of a lightweight, electrodynamic, inertial actuator with integrated velocity sensor for active vibration control of a thin lightly-damped panel, Proceedings of the International Conference on Noise and Vibration Engineering (ISMA), Leuven, Belgium, 20-23 September 2004.

[12] C. Paulitsch, P. Gardonio, S.J. Elliott, Active vibration control using an inertial actuator with internal damping, Journal of the Acoustical Society of America 119 (2006) $2131-2140$.

[13] N. Alujevic, G. Zhao, B. Depraetere, P. Sas, B. Pluymers, W. Desmet, $\mathscr{H}_{2}$ optimal vibration control using inertial actuators and a comparison with tuned mass dampers, Journal of Sound and Vibration 333 (18) (2014) 4073-4083.

[14] P. Gardonio, N. Alujevic, Double panel with skyhook active damping control units for control of sound radiation, Journal of the Acoustical Society of America 128 (3) (2010) 1108-1117.

[15] Z. Qiu, X. Zhang, H. Wu, H. Zhang, Optimal placement and active vibration control for piezoelectric smart flexible cantilever plate, Journal of Sound and Vibration 301 (2007) 521-543.

[16] E. Crawley, J. de Luis, Use of piezoelectric actuators as elements of intelligent structures, AIAA Journal 25 (1987) 1373-1385.

[17] C.R. Fuller, S.J. Elliott, P.A. Nelson, Active Control of Vibration, Academic Press, San Diego, 1986.

[18] T.J. Sutton, S.J. Elliott, M.J. Brennan, K.H. Heron, D.A.C. Jessop, Active isolation of multiple structural waves on a helicopter gearbox support strut, Journal of Sound and Vibration 205 (1) (1997) 81-101.

[19] B. Rebbechi, C. Howard, C. Hansen, Active control of gearbox vibration, Proceedings of the Active control of Sound, Vibration Conference, Fort Lauderdale, 1999, pp. 295-304.

[20] G. Pinte, S. Devos, B. Stallaert, W. Symens, J. Swevers, P. Sas, A piezo-based bearing for the active structural acoustic control of rotating machinery, Journal of Sound and Vibration 329 (2010) 1235-1253.

[21] B. Stallaert, Active Structural Acoustic Source Control of Rotating Machinery PhD thesis, KU Leuven University, 2010.

[22] Y.H. Guan, T.C. Lim, W.S. Shenpard Jr., Experimental study on active vibration control of a gearbox system, Journal of Sound and Vibration 282 (3-5) (2005) 713-733.

[23] M. Li, T.C. Lim, W.S. Shenpard Jr., Y.H. Guan, Experimental active vibration control of gear mesh harmonics in a power recirculation gearbox system using a piezoelectric stack actuator, Smart Materials and Structures 14 (5) (2005) 917-927.

[24] M.H. Chen, M.J. Brennan, Active control of gear vibration using specially configured sensors and actuators, Smart Materials and Structures 9 (3) (2000) $342-350$.

[25] Y.H. Guan, M. Li, T.C. Lim, W.S. Shenpard Jr., Comparative analysis of actuator concepts for active gear pair vibration control, Journal of Sound and Vibration 269 (1-2) (2004) 273-294.

[26] M.J. Brennan, J. Garcia-Bonito, S.J. Elliott, A. David, R.J. Pinnington, Experimental investigation of different actuator technologies for active vibration control, Smart Materials and Structures 8 (1999) 145-153.

[27] C.G. Diaz, Active Structural Acoustic Control Smart Panel With Small Scale Proof Mass Actuators PhD thesis, University of Southampton, England, 2007.

[28] A. Preumont, Vibration Control of Active Structures, Kluwer Academic, London, 2002.

[29] G. Zhao, W. Jacobs, B. Depraetere, N. Alujevic, G. Pinte, P. Sas, Modal analysis of a piezo-based axisymmetric rotational vibration absorber, Proceedings of 5th IOMAC International Operational Modal Analysis Conference, Guimaraes, Portugal (art.nr. Paper Id: no. 229). 
[30] B. Widrow, J. Glover, J. McCool, J. Kaunitz, C. Williams, R. Hearn, J. Zeidler, E. Dong, R. Goodlin, Adaptive noise cancelling: principles and applications, Proceedings of the IEEE 63 (12) (1975) 1692-1716.

[31] J. Glover, Adaptive noise cancelling applied to sinusoidal interferences, IEEE Transactions on Acoustics, Speech and Signal Processing 25 (6) (1977) 484-491.

[32] D. Morgan, C. Sanford, A control theory approach to the stability and transient analysis of the filtered-x Ims adaptive notch filter, IEEE Transactions on Signal Processing 40 (9) (1992) 2341-2346.

[33] S.M. Kuo, D.R. Morgan, Active noise control: a tutorial review, Proceedings of the IEEE 87 (6) (1999) $943-973$.

[34] N. Saito, T. Sone, Influence of modelling error on noise reduction performance of active noise control systems using filtered-x Ims algorithm, Journal of the Acoustical Society of Japan 17 (4) (1998) 195-202. 